Seção Temática: Balanço do Fundeb Volume 10 - $2020 \mid$ n. 19

\title{
Apresentação da Seção Temática: Balanço do Fundeb
}

\author{
Márcia Aparecida Jacomini \\ Universidade Federal de São Paulo (UNIFESP), Guarulhos/SP - Brasil \\ José Marcelino de Rezende Pinto \\ Universidade de São Paulo (USP), São Paulo/SP - Brasil
}

A partir de 1997, com a entrada em vigor do Fundef, que passou a ser um fundo compulsório para todo o Brasil a partir de 1998, o financiamento da educação no Brasil iniciou um novo estágio de organização por meio da subvinculação constitucional de recursos. Este fundo foi substituído pelo Fundeb, com vigência iniciada em 2007 e duração prevista até dezembro de 2020. Tendo em vista o fim desse primeiro ciclo de vigência do Fundeb e o processo de discussão e aprovação do Fundeb permanente, a REVISTA FINEDUCA organizou a Seção Temática: Balanço do Fundeb, publicada em duas partes, uma que sai neste volume de 2020 e outra, na próxima, em 2021.

Quando publicamos o edital para esta seção temática, o novo Fundeb ainda estava em discussão no legislativo. No momento de redação desta apresentação a situação já é outra, com a importante vitória que foi a aprovação da Emenda Constitucional 108/2020 (Novo Fundeb) pelo Congresso Nacional. Entre os avanços cabe destacar:

- garantia do caráter permanente do Fundeb, propiciando a possibilidade de um planejamento efetivo dos gastos educacionais por parte dos secretários de educação, além de retirar o argumento utilizado para a inexistência de planos de carreira atrativos para os profissionais da educação sob a alegação da transitoriedade do fundo;

- incorporação do conceito de Custo Aluno-Qualidade que vincula o financiamento à presença de insumos nas escolas, assegurando condições adequadas de oferta a todas as escolas;

- ampliação da complementação da União ao Fundeb, que hoje é de $10 \%$, para $23 \%$ até 2026;

- proibição de que a União, os estados, o Distrito Federal e os municípios contabilizem os gastos com profissionais da educação aposentados sob a rubrica de manutenção e desenvolvimento do ensino;

- modelo de distribuição de recursos da complementação da União em formato híbrido, que garante os recursos distribuídos pela regra atual para os estados e municípios que já o recebem, mas, em relação aos recursos adicionais, adota o critério de disponibilidade total de recursos educacionais de cada ente (estados, Distrito Federal, ou municípios) de tal forma que municípios pobres em estados considerados mais ricos passarão a receber recursos da complementação, enquanto os municípios com mais recursos educacionais por aluno, pertencentes a estados considerados mais pobres, não receberão essa complementação adicional; 
Apresentação da Seção Temática: Balanço do Fundeb

- vedação do uso, como fonte da complementação da União, de receitas do SalárioEducação, contribuição social que destina recursos para os programas complementares de alimentação escolar, livro didático, dinheiro direto na escola, entre outros.

Com relação a esses avanços, cabe comentar o papel decisivo da Fineduca, por meio de notas técnicas e participação em audiências públicas, sempre em estreita sintonia com a Campanha Nacional pelo Direito à Educação, em especial na garantia do Custo AlunoQualidade (CAQ), do modelo híbrido e da vedação do uso dos recursos do Salário-Educação na complementação federal. Foram três grandes vitórias.

A construção do novo Fundeb, contudo, ainda está a meio caminho. Pontos centrais, como os fatores de ponderação para as etapas e modalidades; destinação de recursos do fundo para instituições privadas de ensino e vinculação de parte da nova complementação federal à educação infantil, precisam ser regulamentados. Particularmente polêmica é a destinação de parte da complementação federal para "redes públicas que, uma vez cumpridas condicionalidades de melhoria de gestão previstas em lei, alcançarem evolução de indicadores a serem definidos, de atendimento e melhoria da aprendizagem com redução das desigualdades, nos termos do sistema nacional de avaliação da educação básica" (art. 212a, inc. V). Embora controverso, tendo em vista o grande risco de negar recursos, exatamente, aos entes federados mais pobres (e com pior desempenho), e que mais deles necessitam, o inciso depende da regulamentação do sistema nacional de avaliação básica (SINAEB), previsto no PNE 2014-24 e que a sociedade brasileira demanda há tempos, o que impede uma implementação açodada. Enquanto não se institucionaliza o SINAEB, seria importante que essa parte da complementação ( $2,5 \%$ do aporte de estados, DF e municípios) seja utilizada para o avanço no atendimento da educação básica, conforme determina a CF e o PNE 2014-2024.

Um aspecto negativo que restou na redação final da EC refere-se à permissão de uso, por parte da União, de recursos advindos da vinculação à Manutenção e Desenvolvimento do Ensino, em um total de até $30 \%$ do valor devido da complementação (art. 212-a, inc. VIII). Trata-se da mesma redação atual do Fundeb, acontece que, como a complementação federal crescerá 2,3 vezes, abre-se a possibilidade de que sejam retirados recursos que hoje são destinados pela União para a educação básica e superior. É um grave risco, sendo necessário encontrar uma forma de impedir essa estratégia por parte do governo federal.

A seção temática inicia-se com o artigo 0 papel do legislativo na discussão de Fundos educacionais. Paulo Sena Martins apresenta uma interessante descrição do papel do legislativo no processo de discussão e aprovação da política de fundos para o financiamento da educação básica; além de descrever o percurso dos projetos de lei relativos aos fundos e indicar os deveres do legislativo na aprovação de leis, no acompanhamento de sua implementação e impactos e na fiscalização do poder executivo.

No processo de discussão do novo Fundeb, a comissão especial da PEC 15/2015, legislatura passada $\left(55^{\mathrm{a}}\right)$, realizou 29 audiências públicas, além de várias reuniões de trabalho em 2017 e 2018. Na atual legislatura (56 $)$, houve uma recomposição da comissão, com manutenção da relatoria e a realização de 16 encontros entre audiência pública e seminários. 
Apresentação da Seção Temática: Balanço do Fundeb

O autor destaca, ainda, a importância da relatoria na construção do texto de projeto de lei e dos consensos possíveis

Já o artigo A política de Fundos e as responsabilidades federativas pela oferta da educação básica, de Nalú Farenzena, tem como objetivo discutir a cooperação federativa na oferta e no financiamento da educação no período de vigência dos fundos a partir da análise dos recursos do Fundeb e da distribuição das matrículas, em suas diferentes etapas e modalidades, entre as dependências administrativas.

O estudo aponta para a municipalização da educação infantil e do ensino fundamental, entre 1996 e 2018, e para o descompasso entre os dados de matrículas e as metas do Plano Nacional de Educação.

Finalmente, a autora analisa a variação do Valor Anual por Aluno (VAA) entre o último ano do Fundef (2006) e 2018, mostrando que houve uma aproximação desse valor entre os estados, em virtude da maior complementação da União no Fundeb e da dinâmica das matrículas. Ressalta, contudo, que a partição da União no Fundeb ainda é limitada.

Com o objetivo de contribuir para um balanço do Fundeb na perspectiva da distribuição de recursos e das despesas com remuneração docente, o artigo Contribuições para um balanço do Fundeb: redistribuição dos recursos, despesas com remuneração e vínculo de trabalho docente, de Márcia Aparecida Jacomini, Marcos Edgar Bassi, Maria Dilnéia Espíndola Fernandes, Rosana Maria Gemaque Rolim e Rubens Barbosa de Camargo, traz importantes dados e análises sobre municipalização de matrículas e de recursos do Fundo, valor aluno/ano e PSPN, uso dos recursos do Fundeb para pagamento dos profissionais da educação de redes estaduais e tipos de vínculos empregatícios de professores destas redes.

Entre as principais conclusões, os autores destacam que, a despeito da redução absoluta das matrículas municipais no período de 2007 a 2019, houve aumento percentual do atendimento municipal em relação ao estadual.

Sobre o valor do custo aluno ano e do valor do PSPN, verificou-se que em 21 estados houve variação positiva, de $10 \%$ em média, tanto nas receitas do Fundeb quanto nas despesas com remuneração dos profissionais do magistério. Por outro lado, observou-se que o número de professores efetivos diminuiu no período mais ou menos na mesma proporção em que cresceu o número de vínculo de contratos temporários.

Os autores chamam a atenção para os impactos negativos da aprovação da Emenda Constitucional n. 95/2016 e para a importância, nesse contexto, da aprovação de um Fundeb permanente com aumento de recursos para educação básica, especialmente por meio da complementação da União.

No texto As múltiplas realidades educacionais dos municípios no contexto do Fundeb, Thiago Alves e José Marcelino de Rezende Pinto realizam um profundo mergulho na diversidade dos municípios brasileiros, indicando suas diferenças em termos de porte populacional, perfil da oferta educacional e indicadores de financiamento.

Valendo-se de dados do IBGE, SIOPE, FINBRA e Censo Escolar, e com vasto ferramental estatístico os autores, buscam responder a duas questões: qual é o nível de variação do gasto por aluno entre os municípios e que características explicam a variação do gasto por aluno? $\mathrm{Na}$ análise, os autores mostram o impacto de fatores como a participação da rede municipal na oferta da educação básica, o peso da receita de impostos que não 
Apresentação da Seção Temática: Balanço do Fundeb

compõem o Fundeb em sua receita, o porte dos municípios e o grau de urbanização. Utilizando a técnica de 'análise de agrupamentos' (cluster analysis), foram identificados seis grupos de municípios similares entre si quanto aos indicadores selecionados.

Por fim, são feitas sugestões para que se melhore a qualidade dos indicadores, fortaleçam-se as instituições que os coletam e disseminam, melhore-se a fiscalização quanto às despesas educacionais e, finalmente, aumente-se o papel equalizador da política de fundos, o que passa pela ampliação da complementação para que se atinja o Custo AlunoQualidade inicial (CAQi).

O artigo Educação Infantil e a política de Fundos: como tem caminhado essa etapa educacional, em especial com a aprovação do Fundeb?, de José Marcelino Rezende Pinto e Bianca Correa, articulando as informações do Censo do IBGE e do Censo Escolar do INEP, com dados de pesquisas de campo, oferece um rico painel quanti-quali da situação da educação infantil no País. Os autores analisam a evolução do atendimento desde a aprovação da LDB e do Fundeb, em 1996, as mudanças na divisão de responsabilidades entre os entes públicos, a participação do setor privado, em particular através dos convênios com o setor público e as diferenças na oferta, considerando as diferentes unidades da federação, localização (urbano x rural), etnia e renda das famílias.

Os resultados indicam uma grande expansão da oferta no período, com o abandono por parte das redes estaduais dessa etapa, consequência direta da política de fundos. No caso das creches, ressalta-se a expansão da rede conveniada no período do Fundeb e grandes diferenças nas taxas de atendimento, quando se consideram as diferentes regiões do país, a localização, etnia e renda das famílias. No caso da faixa da pré-escola, obrigatória desde 2016, os avanços foram significativos, as diferenças nas taxas de atendimento são menores, mas a universalização não foi atingida mesmo entre os segmentos mais ricos da população. Nessa etapa, a presença da jornada em tempo integral é muito pequena, ao contrário das creches, em que essa realidade predomina, mas com grandes variações regionais.

Já os estudos empíricos analisados, com dados de municípios do interior paulista, mostram que, não obstante os avanços no atendimento, identificou-se crianças em fila de espera para vagas em creches; número excessivo de crianças por turma e auxiliares que não são professores contratados para o exercício da docência, indicando que pouco se avançou nos indicadores de qualidade. Permanece a cisão entre cuidado e educação, ainda distante da construção de um modelo sólido de identidade da Educação Infantil. Finalmente, os estudos revistos no artigo apontam para um conjunto de práticas e posturas típicas do Ensino Fundamental, ocorrendo uma "colonização da El" por essa etapa.

O artigo Repercussões do Fundeb no acesso e na oferta da educação infantil: um estudo de caso, de Josielli Teixeira de Paula Costa, Franceline Rodrigues Silva e Daniel Santos Braga, contribui para um balanço do Fundeb em dois aspectos principais, por tratar da educação infantil e por se constituir num estudo de caso envolvendo um município, Ribeirão das Neves, no estado de Minas Gerais.

Foram analisadas três dimensões da oferta e da qualidade da educação infantil no município: expansão da matrícula, infraestrutura das escolas e remuneração docente. Os dados apresentados mostram que a maior parte da receita do município é oriunda das transferências constitucionais e que, em 2009, iniciou-se um período de maior investimento 
Apresentação da Seção Temática: Balanço do Fundeb

na educação infantil. Em relação à oferta, o estudo indica que houve aumento das escolas de educação infantil, tanto creche quanto pré-escola, assim como aumento de matrícula, especialmente na rede municipal de ensino.

Para analisar a remuneração dos professores, os autores utilizaram os dados da Relação Anual de Informações Sociais (RAIS). Até 2014, a remuneração dos professores ficou acima do Piso Salarial Profissional Nacional (PSPN) e abaixo a partir de 2015, fruto da crise econômica, de acordo com os autores. Destacam ainda um predomínio de contratação temporária de professores.

Não obstante o Fundeb ter sido fundamental para a expansão da educação infantil, os autores salientam que ele não é capaz de resolver todos os problemas educacionais, pois ainda constitui desafio para o município de Ribeirão das Neves atender ao menos $50 \%$ das crianças de zero a três anos e universalizar a matrícula das crianças de quatro e cinco anos, conforme preconizado no Plano Nacional de Educação 2014-2024.

Na perspectiva de contribuir com uma avaliação do impacto do Fundeb nas matrículas, o estudo de Rosana Evangelista da Cruz e Aline Kasuko Sonobe, A oferta do Ensino fundamental no contexto do FUNDEB, traz interessantes análises sobre a dinâmica da matrícula no ensino fundamental no período de 2007 a 2019, a taxa de atendimento e a continuidade da municipalização, já verificada na vigência do Fundef.

Partindo do fato de que durante o Fundef, 1996 a 2006, houve um crescimento nas matrículas estaduais e municipais de $1,4 \%$, as autoras analisam os dados do censo escolar e constatam que, entre 2007 a 2009, nesta etapa da educação básica, houve diminuição de $24 \%$ nas matriculas estaduais e municipais, sendo mais intensa no Nordeste (31\%), no Sudeste $(23 \%)$ e no Sul $(22 \%)$. Verificaram, também, que as quedas mais significativas ocorreram nos estados do Nordeste que receberam complementação da União.

Em busca de uma explicação para esse fenômeno, Cruz e Sonobe analisaram a taxa de atendimento escolar e constataram que, embora esse indicador tenha apresentado aumento no período de 2007 a 2015, não alcançou a totalidade da população de seis a 14 anos, mostrando que há potencial de crescimento das matrículas nos anos iniciais e finais do ensino fundamental, destacadamente nas regiões em que houve maior queda na matrícula no período de vigência do Fundeb.

O estudo indica, ainda, que a tendência à municipalização do ensino fundamental verificada durante a vigência do Fundef teve continuidade no Fundeb, chegando a $69 \%$ e sendo mais acentuada nos anos iniciais do ensino fundamental.

Em conclusão, afirmam que "[...] no FUNDEB, de 2007 para 2019, houve crescimento considerável na creche (133\%), moderado na pré-escola (2\%) e queda no Ensino Médio $(15 \%)$ e na EJA (37\%), frustrando as expectativas de que o novo Fundo seria mais inclusivo ao viabilizar o direito à educação para todas as etapas e modalidades do ensino obrigatório no Brasil, o que ocorreu somente na etapa com menor taxa de atendimento, a Educação Infantil".

Outra contribuição para um balanço do Fundeb, do ponto de vista da análise da dinâmica das matrículas e das escolas nas redes estaduais, do Distrito Federal, municipais e privadas, encontra-se no artigo Fundeb: uma avaliação da evolução do número de matrículas e escolas na educação básica, de Nicholas Davies e Alzira Batalha Alcântara. 
Apresentação da Seção Temática: Balanço do Fundeb

Os autores analisaram a evolução das matrículas e das escolas no período de 2007 a 2019 (setor público) e 2006 a 2019 (setor privado), com base nos dados do censo escolar do Inep. Verificaram que houve uma redução nas matrículas estaduais da ordem de $30,2 \%$ e nas municipais de $6,1 \%$, enquanto na rede privada houve um aumento de $24,3 \%$.

Em relação ao número de escolas também houve diminuição no setor público (redes estaduais e municipais) e crescimento na rede privada. $O$ decréscimo das escolas estaduais foi de $8,9 \%$ e, das municipais, de $18,2 \%$. No setor privado houve crescimento do número de escolas em todas as regiões, totalizando um aumento no Brasil de $16,6 \%$.

Embora a maior parte das matrículas da educação básica no período analisado esteja nas redes públicas de ensino, em 2019, 38.334.654 (estaduais e municipais) contra 9.134.785 (privadas), o estudo evidencia que houve um decréscimo da participação da rede estadual de 40,8\% para 32,2\% no total de matrículas da educação básica. As redes municipais, mesmo com diminuição de suas matrículas, aumentaram sua participação de $45,6 \%$ para $48,5 \%$, com ampliação em todas as regiões. A rede privada aumentou sua participação de $13,7 \%$ para $19,2 \%$.

Com base na análise dos dados, os autores consideram que o Fundeb "não contribuiu para manter e desenvolver a educação básica pública, pelos menos no sentido de número de matrículas" nas redes estaduais e municipais.

No artigo Educação Especial e o Fundeb: histórico, balanço e desafios, Marcia Maurilio Souza, Marileide Gonçalves França, Vanessa Dias Bueno de Castro e Rosângela Gavioli Prieto analisam os recursos da educação especial no contexto do Fundeb em âmbito nacional e nas redes estaduais de ensino de São Paulo e Espírito Santo, no período de 2009 a 2019, trazendo importante contribuição sobre o financiamento de uma modalidade de ensino pouco investigada. Foram analisados os censos escolares, os valores aluno/ano do Fundeb e as despesas líquidas do Fundeb, da função educação e da subfunção educação especial.

No período estudado, houve um aumento de 95,5\% na matrícula da educação especial em âmbito nacional, saindo de $1,2 \%$ da matrícula total, em 2009, para 2,4\%, em 2019. As matrículas no estado de São Paulo aumentaram na ordem de $24,3 \%$, no ano de 2019 , em comparação com 2009, e, no Espírito Santo, houve um expressivo aumento de 520,5\%.

Em relação ao valor aluno/ano da educação especial, observou-se para o Brasil um aumento de aproximadamente $60 \%$ e, para os estados de São Paulo e Espírito Santo, o crescimento no período foi de $1 \%$ e $9 \%$, respectivamente.

No que se refere às despesas com a função educação, houve um aumento de $21,3 \%$ no período no estado de São Paulo e 11,2\% para o Espírito Santo. Na subfunção educação especial, os aumentos foram, respectivamente, de 50,5\% e 655,9\%.

Os dados analisados indicam que houve ampliação dos recursos do Fundeb destinados à educação especial, mas as autoras ressaltam que eles podem "[...] ter contemplado outras etapas ou modalidades de ensino, nos respectivos estados, visto que o fundo não prevê a alocação de recursos exclusivos na educação especial". Nesse sentido, de acordo com as autoras, para um acompanhamento mais preciso dos recursos destinados à educação especial faz-se necessário alterar os demonstrativos de despesas.

No artigo $\mathbf{O}$ direito à Educação do Campo no estado do Piauí no contexto do Fundeb, as autoras Jullyane Frazão Santana, Lucineide Barros Medeiros e Marli Clementino 
Apresentação da Seção Temática: Balanço do Fundeb

Gonçalves contribuem com uma importante discussão sobre o financiamento da educação do campo no estado do Piauí. Na primeira parte do texto são retomados aspectos históricos da legislação sobre o direito à educação do campo, destacando a importância dessa discussão no estado, uma vez que, enquanto a proporção da população que vive no campo no Brasil é de $15,28 \%$, no estado do Piauí esse percentual é de 33\%.

As autoras buscam compreender como tem se configurado a oferta da educação básica na zona rural e em que medida o Fundeb contribuiu para a garantia da educação do campo no estado. Informam que o número de escolas que ofertam educação básica no Piauí, no período de 2009 a 2019, diminuiu mais de 50\%; em 2008 eram 4.820 instituições, contra 2.055 em 2019, sendo que a principal queda ocorreu em relação às escolas municipais, que representam o maior número de instituições de ensino rural. Também em relação à matrícula houve uma queda de aproximadamente $40 \%$.

Consideram que a oferta e o fechamento de escolas na zona rural estão relacionados a quatro fatores principais: diminuição da população, decisões governamentais, modelos de gestão adotados e disponibilidade de recursos financeiros. Destacam as autoras que é fundamental garantir um financiamento que considere as demandas da educação do campo e que respeite os modos de viver e trabalhar de tal população.

As autoras Samara de Oliveira Silva, Theresa Maria de Freitas Adrião e Luciene Rodrigues Vasconcelos Borges de Almeida, no artigo Panorama geral do financiamento da educação na rede estadual do Piauí, pós instituição do Fundeb no Brasil, discutem a composição e os montantes de recursos que compõem o Fundeb no estado do Piauí, tendo em vista analisar a destinação de recursos estaduais ao Fundo e o montante recebido.

Além de uma retrospectiva sobre o financiamento da educação pós Constituição Federal de 1988, as autoras apresentam dados demográficos e sociais de um dos estados brasileiros com menor rendimento médio mensal domiciliar per capita.

Entre os achados da pesquisa, verificaram que o Fundo de Participação do Estado (FPE) e o Imposto sobre Circulação de Mercadorias e Serviços (ICMS) contribuíram com 50\% e $44 \%$ respectivamente das receitas orçamentárias do estado do Piauí, em relação ao total de recursos no período de 2007 a 2017. Concluem que, mesmo com a complementação da União, cujo crescimento foi de $289 \%$ no período de 2007 a 2017 , o estado recebeu menos recursos do que destinou ao Fundeb, o que pode ser explicado pelo fato de ele ter uma arrecadação maior do que a dos municípios e pelo intenso processo de municipalização, que ocasionou a queda no número de matrículas na rede estadual.

Desejamos uma boa leitura e que os artigos contribuam para novos questionamentos, pesquisas e, em especial, na regulamentação da nova versão do Fundeb, tarefa urgente do Congresso Nacional e que não pode ser realizada sem a garantia da participação no debate das entidades de pesquisa e de defesa do direito à educação.

Márcia Aparecida Jacomini e José Marcelino de Rezende Pinto

Editores da Revista Fineduca 


\section{Editores do volume 10}

Márcia Aparecida Jacomini - Universidade Federal de São Paulo, Brasil

José Marcelino de Rezende Pinto - Universidade de São Paulo, Brasil

\section{Comitê Editorial}

Nalú Farenzena - Universidade Federal do Rio Grande do Sul, Brasil

Juca Gil - Universidade Federal do Rio Grande do Sul, Brasil

Theresa Adrião - Universidade Estadual de Campinas, Brasil

Ângelo Ricardo de Souza - Universidade Federal do Paraná, Brasil

\section{Conselho Editorial}

\section{Alejandro Morduchowicz}

Universidad Pedagógica, Provincia de Buenos Aires, Argentina

Andréa Barbosa Gouveia

Universidade Federal do Paraná, Brasil

Fernanda Saforcada

Universidade de Buenos Aires, Argentina

Jacques Velloso

Universidade de Brasília, Brasil

João Monlevade

Senado Federal, Brasil

Jorge Abrahão de Castro

Instituto de Pesquisa Econômica Aplicada / IPEA, Brasil

Lisete Regina Gomes Arelaro

Universidade de São Paulo, Brasil

Luis Carlos Sales

Universidade Federal do Piauí, Brasil

Luiz de Sousa Junior

Universidade Federal da Paraíba, Brasil

Luiz Fernandes Dourado

Universidade Federal de Goiás, Brasil

Magna França

Universidade Federal do Rio Grande do Norte, Brasil

Marcos Edgar Bassi

Universidade Federal de Santa Catarina, Brasil

Maria Angélica Pedra Minhoto

Universidade Federal de São Paulo, Brasil

Maria Beatriz Luce

Universidade Federal do Rio Grande do Sul, Brasil

Maria Dilnéia Espíndola Fernandes

Universidade Federal de Mato Grosso do Sul, Brasil

Nelson Cardoso do Amaral

Universidade Federal de Goiás, Brasil

Nicholas Davies

Universidade Federal Fluminense, Brasil

Robert E. Verhine

Universidade Federal da Bahia, Brasil

Romualdo Portela de Oliveira

Universidade de São Paulo, Brasil

Rosana Gemaque Rolim

Universidade Federal do Pará, Brasil

Rubens Barbosa de Camargo

Universidade de São Paulo, Brasil

Theresa Adrião

Universidade Estadual de Campinas, Brasil

Tristan McCowan

University of London, Reino Unido

Vera Jacob

Universidade Federal do Pará, Brasil

Vera Peroni

Universidade Federal do Rio Grande do Sul, Brasil

Vitor Henrique Paro

Universidade de São Paulo, Brasil

\section{Equipe editorial}

Apoio ao Comitê Editorial: Caio Cabral da Silva

Diagramação, Revisão de português e normalização: Edson Leonel de Oliveira

Revisão de inglês: Sabrina Ferreira

Fineduca - Revista de Financiamento da Educação

Associação Nacional de Pesquisa em

Financiamento da Educação

e-mail: revista.fineduca@gmail.com | site: http://seer.ufrgs.br/fineduca 